\title{
Thickness effects in high-angle annular dark-field imaging of interfaces
}

\author{
D.O. Klenov and S. Stemmer
}

Materials Department, University of California, Santa Barbara, CA

93106-5050

In recent years, high-angle annular dark field (HAADF) imaging in scanning transmission electron microscopy (STEM) has become widely recognized as a technique that provides chemically sensitive atomic resolution structure images that are generally more directly interpretable than conventional high-resolution transmission electron microscope (HRTEM) images. In contrast, the interpretation of HAADF image intensities in terms of the occupancy of the atomic columns, i.e. their average atomic number, is less well understood. Coherence along the atomic columns and the influence of probe channeling, i.e. the tendency of the probe to focus along the atomic columns, may cause the image contrast to become a function of sample thickness. Very thin samples are often practically difficult to realize. For example, samples may contain interfaces between materials with very different ion-milling rates.

In this presentation, we discuss the influence of sample thickness on HAADF image contrast for a wide range of atomic numbers $(Z)$ for the particular case where the spacing between the columns is significantly larger than the incident probe. Model systems that will be discussed include epitaxial $\mathrm{PbTiO}_{3}$ thin films on $\mathrm{SrTiO}_{3}$ substrates, epitaxial ErAs layers on (In,Ga)As [1] and epitaxial $\mathrm{SrTiO}_{3}$ films on Pt electrodes. These model systems allowed for the quantitative analysis of image contrast for a wide range of atomic numbers and crystal structures. Quantitative evaluation of image contrast as a function of thickness also requires precise knowledge of the electron optical parameters, such as focus and detector inner angle, and the influence of these parameters on the HAADF image contrast will also be discussed.

Sample thicknesses ranging from $\sim 10 \mathrm{~nm}$ to more than $400 \mathrm{~nm}$ were investigated (Fig. 1). The image contrast was relatively insensitive to changes in inner angle. Even for large thicknesses and heavily scattering elements, such as $\mathrm{Pb}$, the image contrast reflected the atomic number, no contrast reversals were observed, and light columns were not "swamped out" by neighboring heavy columns. The main impact of sample thickness was a rapid increase in a background intensity that contributed equally to the intensities of the atomic columns and the channels between them. The background intensity and its increase with thickness reflected the average atomic number of the crystal. Subtraction of the background intensity allowed for a quantitative interpretation of image contrast in terms of atomic numbers and comparison with multislice image simulations. For analysis of heterointerfaces [1] in terms of atom column occupancy, very thin TEM samples are required to minimize the contribution of the background that cannot be calculated or experimentally extracted at interfaces (Fig. 2).

The authors thank the DOE Office of Basic Energy Sciences for support of this research (grant \#DE-FG03-02ER45994), Prof. Paul McIntyre's group for the $\mathrm{SrTiO}_{3} / \mathrm{PbTiO}_{3}$ samples, and Prof. Art Gossard's group for the ErAs/(In,Ga)As samples.

\section{References}

[1] D. O. Klenov, J. M. Zide, J. D. Zimmerman, A. C. Gossard, S. Stemmer, Interface atomic structure of epitaxial ErAs layers on (001) $\mathrm{In}_{0.53} G a_{0.47} A s$ and GaAs, Appl. Phys. Lett. 86, 241901 (2005). 

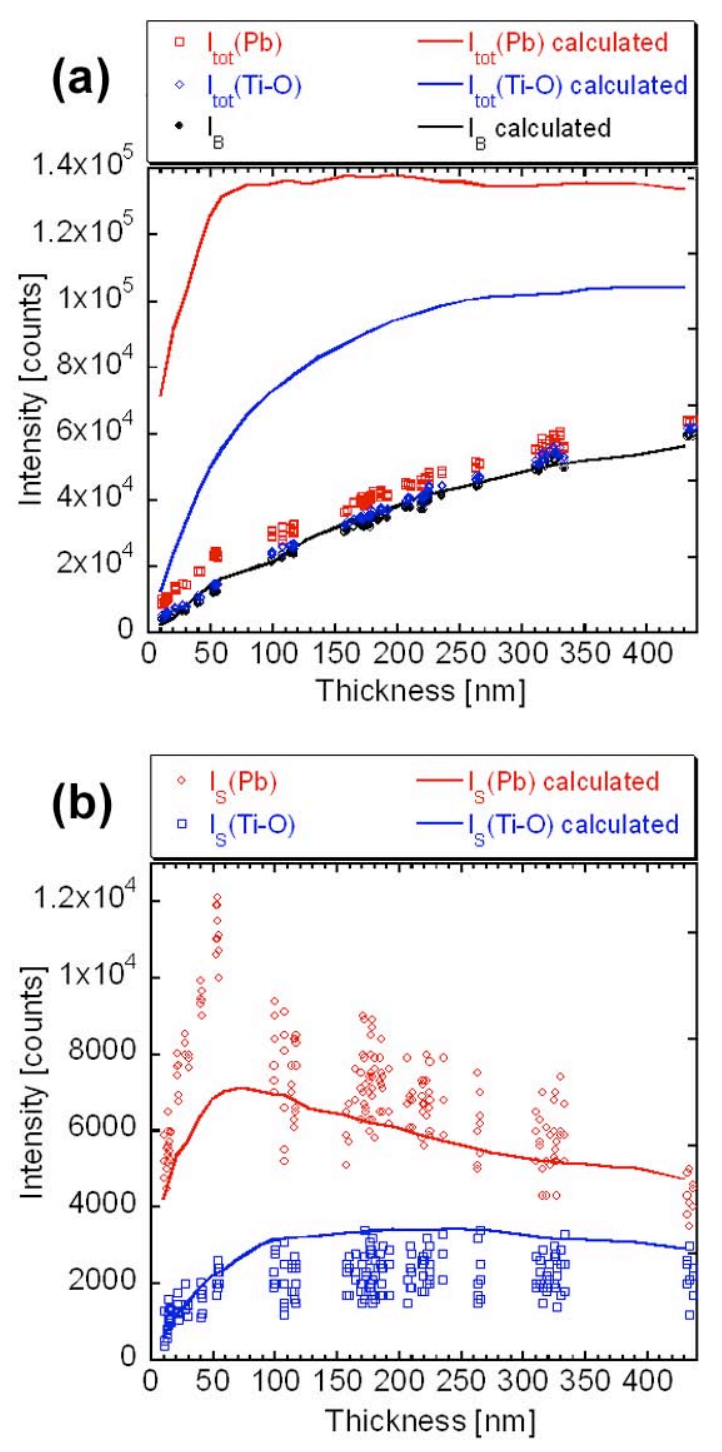

Fig. 1: (a) Comparison of experimental (symbols) and simulated (lines) $\mathrm{PbTiO}_{3}$ atom column and background intensities as a function of sample thickness, scaled so that the calculated values for $I_{B}$ at the largest thickness corresponded to the experimental value. (b) Comparison of experimental (symbols) and simulated (lines) $\mathrm{PbTiO}_{3}$ atom column intensities after subtraction of the background intensity as a function of sample thickness, scaled so that the calculated values at the largest thickness corresponded to the experimental value.

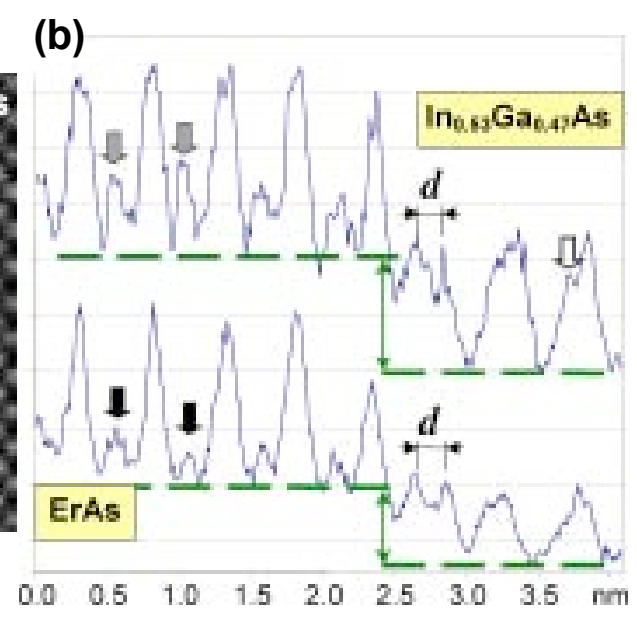

Fig. 2: (a) HAADF image of a ErAs $/ \mathrm{In}_{0.53} \mathrm{Ga}_{0.47} \mathrm{As}$ interface along โ1 110$\rfloor$. The overlay shows the positions of Er columns in red, As columns in blue and (In,Ga) yellow. Arrows mark the last row of (Ga-In) in the semiconductor. Intensity profiles across the interfaces from regions with different thickness. The background (thick dashed lines) is greater in the ErAs relative to the $\mathrm{In}_{0.53} \mathrm{Ga}_{0.47} \mathrm{As}$. The block arrows indicate the As-columns. The column spacing labeled " $d$ " marks the spacing between the last In-Ga column and the As column in the first ErAs layer. 